\title{
On the Solutions of Nonlinear Higher-Order Boundary Value Problems by Using Differential Transformation Method and Adomian Decomposition Method
}

\author{
Che Haziqah Che Hussin and Adem Kiliçman \\ Department of Mathematics, University Putra Malaysia, 43400 UPM Serdang, Selangor, Malaysia
}

Correspondence should be addressed to Adem Kiliçman, akilicman@putra.upm.edu.my

Received 8 November 2010; Accepted 7 February 2011

Academic Editor: Alexei Mailybaev

Copyright (C) 2011 C. H. Che Hussin and A. Kiliçman. This is an open access article distributed under the Creative Commons Attribution License, which permits unrestricted use, distribution, and reproduction in any medium, provided the original work is properly cited.

\begin{abstract}
We study higher-order boundary value problems (HOBVP) for higher-order nonlinear differential equation. We make comparison among differential transformation method (DTM), Adomian decomposition method (ADM), and exact solutions. We provide several examples in order to compare our results. We extend and prove a theorem for nonlinear differential equations by using the DTM. The numerical examples show that the DTM is a good method compared to the ADM since it is effective, uses less time in computation, easy to implement and achieve high accuracy. In addition, DTM has many advantages compared to ADM since the calculation of Adomian polynomial is tedious. From the numerical results, DTM is suitable to apply for nonlinear problems.
\end{abstract}

\section{Introduction}

Recently, many researchers use ADM to approximate numerical solutions. In [1], Wazwaz proposed a modification of ADM method in series solution to accelerate its rapid convergence, and, in [2], Wazwaz also presented several numerical examples of higher-order boundary value problems for first-order linear equation and second-order nonlinear equation by applying modified decomposition method.

In addition, Wazwaz [3,4] provided first-order linear and second-order nonlinear problems to solve fifth-order and sixth-order boundary value problems by the modified decomposition method. Later, Meštrović [5] solved eight-order boundary value problems for first-order linear and second-order nonlinear boundary value problems. Similarly, in [6], Hosseini and Jafari used Adomian decomposition method to solve high-order and system of nonlinear differential equations. 
The Adomian decomposition method is widely used in applied science to compute the series solution accurately because it provides rapid convergent series to solve the problem. The big application of ADM in research area is stochastic and deterministic problems involving differential, integrodifferential, integral, differential delay, and systems of such equations; for example, see [3, 7]. For example, we use the same method for solving several different problems, such as, in calculus of variations, see [8], for eikonal partial differential equation, see [9], for the Fitzhugh-Nagumo equation which models the transmission of nerve impulses, see [10], for linear and nonlinear systems of Volterra functional equations using Adomian-Pade technique, see [11], for coupled Burgers equations by using Adomian-Pade technique see [12], for solution of a nonlinear time-delay model in biology by using semianalytical approaches, see [13], for solving the pantograph equation of order $m$, see [14], and for nonclassic problem for one-dimensional hyperbolic equation by using the decomposition procedure, see [15].

Further, Ray and Bera in [16] used ADM to solve analytical solution of a fractional diffusion equation. They performed the explicit solution of the equation in the closed form by using initial value problem.

In [17], Ayaz investigated initial value problem of partial differential equation (PDE) to solve two-dimensional differential transformation method, and we compare the results with Adomian decomposition method. The results show that the solutions of the present method are exactly the same as the decomposition method, but the calculation of DTM is simple and reduces the difficulty of calculations.

Ayaz in [18] performed two- and three-dimensional differential transformation methods to find exact solutions of linear and nonlinear partial differential equations. Results are compared to decomposition method, and DTM has less computational effort. After that, Ertürk and Momani in [19] presented numerical solution by comparing the differential transformation method (DTM) and Adomian decomposition method (ADM) for solving linear and nonlinear fourth-order boundary value problems and proved that DTM is very accurate and efficient in numerical solution.

Recently, Arikoglu and Ozkol solved fractional differential equations by using differential transform method. They applied fractional differential equations to various types of problems such as the Bagley-Torvik, Ricatti, and composite fractional oscillation equations; see [20].

In this study, we make comparison among differential transformation method, Adomian decomposition method and exact solutions. We prove that DTM is more powerful technique than ADM and can be applied to nonlinear problems easily.

\section{Differential Transformation Method}

Suppose that the function $y(x)$ is continuously differentiable in the interval $\left(x_{0}-r, x_{0}+r\right)$ for $r>0$, then we have the following definition.

Definition 2.1. The differential transform of the function $y(x)$ for the $k$ th derivative is defined as follows:

$$
Y(k)=\frac{1}{k !}\left[\frac{d^{k} y(x)}{d x^{k}}\right]_{x=x_{0}}
$$


where $y(x)$ is the original function and $Y(k)$ is the transformed function. The inverse differential transform of $Y(k)$ is defined as

$$
y(x)=\sum_{k=0}^{\infty}\left(x-x_{0}\right)^{k} Y(k)
$$

Note that, the substitution of (2.1) into (2.2) yields the following equation:

$$
y(x)=\sum_{k=0}^{\infty}\left(x-x_{0}\right)^{k} \frac{1}{k !}\left[\frac{d^{k} y(x)}{d x^{k}}\right]_{x=x_{0}}
$$

which is the Taylor's series for $y(x)$ at $x=x_{0}$.

The following theorems are operations of differential transforms.

Theorem 2.2. If $t(x)=r(x) \pm p(x)$, then $T(k)=R(k) \pm P(k)$.

Theorem 2.3. If $t(x)=\alpha r(x)$, then $T(k)=\alpha R(k)$.

Theorem 2.4. If $t(x)=d r(x) / d x$, then $T(k)=(k+1) R(k+1)$.

Theorem 2.5. If $t(x)=d^{2} r(x) / d x^{2}$, then $T(k)=(k+1)(k+2) R(k+2)$.

Theorem 2.6. If $t(x)=\left(d^{b} r(x)\right) / d x^{b}$, then $T(k)=(k+1)(k+2) \cdots(k+b) R(k+b)$.

Theorem 2.7. If $t(x)=r(x) p(x)$, then $T(k)=\sum_{l=0}^{k} P(l) R(k-l)$.

Theorem 2.8. If $t(x)=x^{b}$, then $T(k)=\delta(k-b)$, where

$$
\delta(k-b)= \begin{cases}1, & \text { if } k=b, \\ 0, & \text { if } k \neq b .\end{cases}
$$

Theorem 2.9. If $t(x)=\exp (\lambda x)$, then $T(k)=\lambda^{k} / k$ !.

Theorem 2.10. If $t(x)=(1+x)^{b}$, then $T(k)=b(b-1) \cdots(b-k+1) / k$ !

Theorem 2.11. If $t(x)=\sin (j x+\alpha)$, then $T(k)=\left(j^{k} / k !\right) \sin ((\pi k / 2)+\alpha)$.

Theorem 2.12. If $t(x)=\cos (j x+\alpha)$, then $T(k)=\left(j^{k} / k !\right) \cos ((\pi k / 2)+\alpha)$.

See the details in [21]. 


\section{General Differential Transformation}

Now we prove the following generalized theorem.

Theorem 3.1. The general differential transformation for nonlinear nth-order BVPs, $y^{(n)}(x)=$ $e^{-x} y^{m}(x)$, is given by

$$
Y(n+k)=\frac{k !}{(n+k) !}\left[\sum_{k_{m}=0}^{k} \sum_{k_{m-1}=0}^{k_{m}} \cdots \sum_{k_{1}=0}^{k_{2}}\left(\frac{(-1)^{k_{1}}}{k_{1} !}\right)\left(\left[\prod_{i=2}^{m} Y\left(k_{i}-k_{i-1}\right)\right] Y\left(k-k_{m}\right)\right)\right] .
$$

Proof. We prove this theorem by induction method. First of all, we prove the generalization of the differential equation $e^{-x} y^{m}(x)$. Let $m=2$, then

$$
y^{(n)}(x)=e^{-x} y^{m}(x)
$$

For $k \geq 1$, we have

$$
\begin{gathered}
y^{(n+k)}(x)=\sum_{k_{2}=0}^{k} \sum_{k_{1}=0}^{k_{2}} \frac{k !(-1)^{k_{1}} e^{-x}}{k_{1} !\left(k_{2}-k_{1}\right) !\left(k-k_{2}\right) !} y^{\left(k_{2}-k_{1}\right)}(x) y^{\left(k-k_{2}\right)}(x), \\
\left.y^{(n+k)}(x)\right|_{x=0}=\sum_{k_{2}=0}^{k} \sum_{k_{1}=0}^{k_{2}} \frac{k !(-1)^{k_{1}}}{k_{1} !\left(k_{2}-k_{1}\right) !\left(k-k_{2}\right) !} y^{\left(k_{2}-k_{1}\right)}(x) y^{\left(k-k_{2}\right)}(x) .
\end{gathered}
$$

By definition, we have

$$
\begin{gathered}
(n+k) ! Y(n+k)=\sum_{k_{2}=0}^{k} \sum_{k_{1}=0}^{k_{2}} \frac{k !(-1)^{k_{1}}}{k_{1} !\left(k_{2}-k_{1}\right) !\left(k-k_{2}\right) !}\left(k_{2}-k_{1}\right) ! Y\left(k_{2}-k_{1}\right)\left(k-k_{2}\right) ! Y\left(k-k_{2}\right), \\
(n+k) ! Y(n+k)=\sum_{k_{2}=0}^{k} \sum_{k_{1}=0}^{k_{2}} \frac{k !(-1)^{k_{1}}}{k_{1} !} Y\left(k_{2}-k_{1}\right) Y\left(k-k_{2}\right), \\
Y(n+k)=\frac{k !}{(n+k) !} \sum_{k_{2}=0}^{k} \sum_{k_{1}=0}^{k_{2}} \frac{(-1)^{k_{1}}}{k_{1} !} Y\left(k_{2}-k_{1}\right) Y\left(k-k_{2}\right) .
\end{gathered}
$$

From (3.1), for $m=2$, we have

$$
Y(n+k)=\frac{k !}{(n+k) !} \sum_{k_{2}=0}^{k} \sum_{k_{1}=0}^{k_{2}} \frac{(-1)^{k_{1}}}{k_{1} !} Y\left(k_{2}-k_{1}\right) Y\left(k-k_{2}\right)
$$


This implies that the theorem holds for $m=2$. Now assume that for $m=p$, the DT is as follows:

$$
\begin{aligned}
Y(n+k)= & \frac{k !}{(n+k) !}\left[\sum_{k_{p}=0}^{k} \sum_{k_{p-1}=0}^{k_{p}} \cdots \sum_{k_{1}=0}^{k_{2}}\left(\frac{(-1)^{k_{1}}}{k_{1} !}\right)\left(\left[\prod_{i=2}^{p} Y\left(k_{i}-k_{i-1}\right)\right] Y\left(k-k_{p}\right)\right)\right], \\
Y(n+k)= & \frac{k !}{(n+k) !} \\
& \times\left[\sum_{k_{p}=0}^{k} \sum_{k_{p-1}=0}^{k_{p}} \cdots \sum_{k_{1}=0}^{k_{2}}\left(\frac{(-1)^{k_{1}}}{k_{1} !}\right)\left(Y\left(k_{2}-k_{1}\right) Y\left(k_{3}-k_{2}\right) \cdots\left(k_{p}-k_{p-1}\right) Y\left(k-k_{p}\right)\right)\right] .
\end{aligned}
$$

Note that $m=p$ we have

$$
y^{(n)}(x)=e^{-x} y^{p}(x)
$$

For $k \geq 1$, we have

$$
\left.y^{(n+k)}(x)\right|_{x=0}=\sum_{k_{p}=0}^{k} \sum_{k_{p-1}=0}^{k_{p}} \cdots \sum_{k_{1}=0}^{k_{2}} \frac{k !(-1)^{k_{1}} y^{\left(k_{2}-k_{1}\right)}(x) y^{\left(k_{3}-k_{2}\right)}(x) \cdots y^{\left(k_{p}-k_{p-1}\right)}(x) y^{\left(k-k_{p}\right)}(x)}{k_{1} !\left(k_{2}-k_{1}\right) !\left(k_{3}-k_{2}\right) ! \cdots\left(k_{p}-k_{p-1}\right) !\left(k-k_{p}\right) !}
$$

Thus, for $m=p+1$, we have

$$
y^{(n)}(x)=e^{-x} y^{p}(x) y(x)
$$

For $k \geq 1$, we have

$$
\left.y^{(n+k)}(x)\right|_{x=0}=\sum_{k_{p+1}=0}^{k} \sum_{k_{p}=0}^{k_{p+1}} \cdots \sum_{k_{1}=0}^{k_{2}} \frac{k !(-1)^{k_{1}} y^{\left(k_{2}-k_{1}\right)}(x) y^{\left(k_{3}-k_{2}\right)}(x) \cdots y^{\left(k_{p+1}-k_{p}\right)}(x) y^{\left(k-k_{p+1}\right)}(x)}{k_{1} !\left(k_{2}-k_{1}\right) !\left(k_{3}-k_{2}\right) ! \cdots\left(k_{p+1}-k\right) !\left(k-k_{p+1}\right) !}
$$

By definition, we have

$$
\begin{gathered}
(n+k) ! \Upsilon(n+k)=\sum_{k_{p+1}=0}^{k} \cdots \sum_{k_{1}=0}^{k_{2}} \frac{k !(-1)^{k_{1}}\left(k_{2}-k_{1}\right) ! Y\left(k_{2}-k_{1}\right) \cdots\left(k-k_{p+1}\right) ! Y\left(k-k_{p+1}\right)}{k_{1} !\left(k_{2}-k_{1}\right) ! \cdots\left(k-k_{p+1}\right) !} \\
Y(n+k)=\frac{k !}{(n+k) !} \sum_{k_{p+1}=0}^{k} \sum_{k_{p}=0}^{k_{p+1}} \cdots \sum_{k_{1}=0}^{k_{2}} \frac{(-1)^{k_{1}}}{k_{1}} Y\left(k_{2}-k_{1}\right) Y\left(k_{3}-k_{2}\right) \cdots\left(k_{p+1}-k_{p}\right) Y\left(k-k_{p+1}\right) .
\end{gathered}
$$


From (3.1), for $m=p+1$, we have

$$
\begin{gathered}
Y(n+k)=\frac{k !}{(n+k) !}\left[\sum_{k_{p+1}=0}^{k} \sum_{k_{p}=0}^{k_{p+1}} \cdots \sum_{k_{1}=0}^{k_{2}}\left(\frac{(-1)^{k_{1}}}{k_{1} !}\right)\left(\left[\prod_{i=2}^{p+1} Y\left(k_{i}-k_{i-1}\right)\right] Y\left(k-k_{p+1}\right)\right)\right] \\
Y(n+k)=\frac{k !}{(n+k) !} \sum_{k_{p+1}=0}^{k} \sum_{k_{p}=0}^{k_{p+1}} \cdots \sum_{k_{1}=0}^{k_{2}} \frac{(-1)^{k_{1}}}{k_{1}} Y\left(k_{2}-k_{1}\right) Y\left(k_{3}-k_{2}\right) \cdots\left(k_{p+1}-k_{p}\right) Y\left(k-k_{p+1}\right) .
\end{gathered}
$$

This implies that the theorem holds for $m=p+1$. Now, we prove the generalization of the BVPs,

$$
y^{(n)}(x)=e^{-x} y^{m}(x)
$$

For that purpose, we fixed $m=2$.

For $n=1$ and $k \geq 1$, we have

$$
\left.y^{(1+k)}(x)\right|_{x=0}=\sum_{k_{2}=0}^{k} \sum_{k_{1}=0}^{k_{2}} \frac{k !(-1)^{k_{1}}}{k_{1} !\left(k_{2}-k_{1}\right) !\left(k-k_{2}\right) !} y^{\left(k_{2}-k_{1}\right)}(x) y^{\left(k-k_{2}\right)}(x) .
$$

By definition, we have

$$
\begin{gathered}
(n+k) ! Y(n+k)=\sum_{k_{2}=0}^{k} \sum_{k_{1}=0}^{k_{2}} \frac{k !(-1)^{k_{1}}}{k_{1} !\left(k_{2}-k_{1}\right) !\left(k-k_{2}\right) !}\left(k_{2}-k_{1}\right) ! Y\left(k_{2}-k_{1}\right)\left(k-k_{2}\right) ! Y\left(k-k_{2}\right), \\
Y(1+k)=\frac{k !}{(1+k) !} \sum_{k_{2}=0}^{k} \sum_{k_{1}=0}^{k_{2}} \frac{(-1)^{k_{1}}}{k_{1} !} Y\left(k_{2}-k_{1}\right) Y\left(k-k_{2}\right) .
\end{gathered}
$$

From (3.1), for $n=1$, we have

$$
\begin{gathered}
Y(1+k)=\frac{k !}{(1+k) !}\left[\sum_{k_{2}=0}^{k} \sum_{k_{1}=0}^{k_{2}}\left(\frac{(-1)^{k_{1}}}{k_{1} !}\right)\left(\left[\prod_{i=2}^{2} Y\left(k_{i-1}-k_{i-2}\right)\right] Y\left(k-k_{2}\right)\right)\right], \\
Y(1+k)=\frac{k !}{(1+k) !} \sum_{k_{2}=0}^{k} \sum_{k_{1}=0}^{k_{2}} \frac{(-1)^{k_{1}}}{k_{1} !} Y\left(k_{2}-k_{1}\right) Y\left(k-k_{2}\right) .
\end{gathered}
$$

Now that is the required result; thus, the theorem holds for $n=1$.

Now assume that for $n=q$, the DT is as follows:

$$
Y(q+k)=\frac{k !}{(q+k) !} \sum_{k_{2}=0}^{k} \sum_{k_{1}=0}^{k_{2}} \frac{(-1)^{k_{1}}}{k_{1} !} Y\left(k_{2}-k_{1}\right) Y\left(k-k_{2}\right)
$$


For $k \geq 1$, we have

$$
\left.y^{(q+k)}(x)\right|_{x=0}=\sum_{k_{2}=0}^{k} \sum_{k_{1}=0}^{k_{2}} \frac{k !(-1)^{k_{1}}}{k_{1} !\left(k_{2}-k_{1}\right) !\left(k-k_{2}\right) !} y^{\left(k_{2}-k_{1}\right)}(x) y^{\left(k-k_{2}\right)}(x)
$$

Thus, for $n=q+1$, we have

$$
y^{(q+1)}(x)=(y(x))^{2} e^{(-x)} .
$$

For $k \geq 1$, we have

$$
\begin{gathered}
\left.y^{(q+1+k)}(x)\right|_{x=0}=\sum_{k_{2}=0}^{k} \sum_{k_{1}=0}^{k_{2}} \frac{k !(-1)^{k_{1}}}{k_{1} !\left(k_{2}-k_{1}\right) !\left(k-k_{2}\right) !} y^{\left(k_{2}-k_{1}\right)}(x) y^{\left(k-k_{2}\right)}(x), \\
(q+1+k) ! Y(q+1+k)=\sum_{k_{2}=0}^{k} \sum_{k_{1}=0}^{k_{2}} \frac{k !(-1)^{k_{1}}}{k_{1} !\left(k_{2}-k_{1}\right) !\left(k-k_{2}\right) !} y^{\left(k_{2}-k_{1}\right)}(x) y^{\left(k-k_{2}\right)}(x), \\
Y(q+1+k)=\frac{k !}{(q+1+k) !} \sum_{k_{2}=0}^{k} \sum_{k_{1}=0}^{k_{2}} \frac{(-1)^{k_{1}}}{k_{1} !} Y\left(k_{2}-k_{1}\right) Y\left(k-k_{2}\right) .
\end{gathered}
$$

From (3.1), for $n=q+1$, we have

$$
\begin{gathered}
Y(q+1+k)=\frac{k !}{(q+1+k) !}\left[\sum_{k_{2}=0}^{k} \sum_{k_{1}=0}^{k_{2}}\left(\frac{(-1)^{k_{1}}}{k_{1} !}\right)\left(\left[\prod_{i=2}^{2} Y\left(k_{i-1}-k_{i-2}\right)\right] Y\left(k-k_{2}\right)\right)\right], \\
Y(q+1+k)=\frac{k !}{(q+1+k) !} \sum_{k_{2}=0}^{k} \sum_{k_{1}=0}^{k_{2}} \frac{(-1)^{k_{1}}}{k_{1} !} Y\left(k_{2}-k_{1}\right) Y\left(k-k_{2}\right) .
\end{gathered}
$$

Note that the theorem holds for $n=q+1$.

Example 3.2. By using DTM, we solve the following nonlinear equation of order three for fifth-order BVP:

$$
y^{(5)}(x)=e^{-x}(y(x))^{3}, \quad 0<x<1
$$

subject to the boundary conditions

$$
y(0)=1, \quad y^{\prime}(0)=\frac{1}{2}, \quad y^{\prime \prime}(0)=\frac{1}{4}, \quad y(1)=e^{1 / 2}, \quad y^{\prime}(1)=\left(\frac{1}{2}\right) e^{1 / 2}
$$


By using Theorem 3.1, (3.22) is transformed to the following equation:

$$
Y(r+5)=\frac{r !}{(r+5) !} \sum_{l}^{r} \sum_{s}^{l} \sum_{p}^{s} \frac{(-1)^{p}}{p !} \Upsilon(s-p) Y(l-s) Y(r-l)
$$

By using (2.1) at $x=0$, we obtain the following transformed boundary conditions:

$$
Y(0)=0, \quad Y(1)=\frac{1}{2}, \quad Y(2)=\frac{1}{8}
$$

where $t=y^{\prime \prime \prime}(0) / 3 !=\Upsilon(3)$ and $w=y^{(4)}(0) / 4 !=Y(4)$.

By using the transformed equation (3.24) together with the transformed boundary conditions (3.25), we can easily solve $Y(r)$, for $r \geq 5$. The constants $t$ and $w$ can be evaluated by using boundary conditions (3.23) at $x=1$ :

$$
\begin{gathered}
\sum_{r=0}^{21} Y(r)=e^{1 / 2}, \\
\sum_{r=0}^{21} r Y(r)=\left(\frac{1}{2}\right) e^{1 / 2} .
\end{gathered}
$$

These equations give $t=0.03041272159$ and $w=-0.01577358145$.

Finally, the following series solution can be formed by applying the inverse transformation in (2.2) up to $N=14$ :

$$
\begin{aligned}
y(x)= & 1.0+0.5 x+0.125 x^{2}+0.304127215 \times 10^{-1} x^{3} \\
& -0.1577358145 \times 10^{-1} x^{4}+0.833333333 \times 10^{-2} x^{5} \\
& +0.694444444 \times 10^{-3} x^{6}+0.4960317460 \times 10^{-4} x^{7} \\
& +0.737671102 \times 10^{-5} x^{8}-0.3474145349 \times 10^{-5} x^{9} \\
& +8.094962522 \times 10^{-7} x^{10}+4.176090542 \times 10^{-8} x^{11} \\
& -1.102928451 \times 10^{-8} x^{12}+1.334963335 \times 10^{-8} x^{13} \\
& -7.232116854 \times 10^{-9} x^{14} .
\end{aligned}
$$

Example 3.3. Next, we solve the following fourth-order nonlinear for fifth-order BVP by using DTM:

$$
y^{(5)}(x)=e^{x}(y(x))^{4}, \quad 0<x<1
$$


subject to the boundary conditions

$$
y(0)=1, \quad y^{\prime}(0)=-\frac{1}{3}, \quad y^{\prime \prime}(0)=\frac{1}{9}, \quad y(1)=e^{-1 / 3}, \quad y^{\prime}(1)=\left(-\frac{1}{3}\right) e^{-1 / 3} .
$$

By using Theorem 3.1, (3.28) is transformed to the following equation:

$$
Y(r+5)=\frac{r !}{(r+5) !} \sum_{l}^{r} \sum_{s}^{l} \sum_{p}^{s} \sum_{j}^{p} \frac{(1)^{j}}{j !} \Upsilon(p-j) Y(s-p) Y(l-s) Y(r-l) .
$$

By using (2.1) at $x=0$, we obtain the following transformed boundary conditions:

$$
Y(0)=1, \quad Y(1)=\frac{-1}{3}, \quad Y(2)=\frac{1}{18},
$$

where $t=y^{\prime \prime \prime}(0) / 3 !=\Upsilon(3)$ and $w=y^{(4)}(0) / 4 !=\Upsilon(4)$.

By using the transformed equation (3.30) together with the transformed boundary conditions (3.31), we can easily solve $Y(r)$, for $r \geq 5$. The constants $t$ and $w$ can be evaluated by using boundary conditions (3.29) at $x=1$ :

$$
\begin{gathered}
\sum_{k=0}^{20} Y(r)=e^{-1 / 3}, \\
\sum_{k=0}^{20} r Y(r)=\left(-\frac{1}{3}\right) e^{-1 / 3} .
\end{gathered}
$$

These equations give $t=0.001331809417$ and $w=-0.01491571112$.

Finally, the following series solution can be formed by applying the inverse transformation in (2.2) up to $N=20$ :

$$
\begin{aligned}
y(x)= & 1.0-0.3333333333 x+0.05555555556 x^{2}+0.001331809417 x^{3} \\
& -0.1491571112 \times 10^{-1} x^{4}+0.008333333333 x^{5}-0.462962963 \times 10^{-3} x^{6} \\
& +0.220458553 \times 10^{-4} x^{7}+0.354847562 \times 10^{-5} x^{8} \\
& -0.404801946 \times 10^{-5} x^{9}+0.110569490 \times 10^{-5} x^{10} \\
& -2.741069465 \times 10^{-8} x^{11}-1.250500836 \times 10^{-8} x^{12} \\
& -1.134323528 \times 10^{-8} x^{13}-3.968854379 \times 10^{-9} x^{14} \\
& +2.378413332 \times 10^{-10} x^{15}+1.215436605 \times 10^{-10} x^{16} \\
& -2.331970237 \times 10^{-11} x^{17}+1.269686222 \times 10^{-11} x^{18} .
\end{aligned}
$$


Example 3.4. We perform the following third order of nonlinear equation for sixth-order BVP by using DTM:

$$
y^{(6)}(x)=e^{x}(y(x))^{3}, \quad 0<x<1
$$

subject to the boundary conditions

$$
\begin{gathered}
y(0)=1, \quad y^{\prime}(0)=-\frac{1}{2}, \quad y^{\prime \prime}(0)=\frac{1}{4}, \quad y(1)=e^{-1 / 2}, \\
y^{\prime}(1)=\left(-\frac{1}{2}\right) e^{-1 / 2}, \quad y^{\prime \prime}(1)=\left(\frac{1}{4}\right) e^{-1 / 2} .
\end{gathered}
$$

By using Theorem 3.1, (3.34) is transformed to the following equation:

$$
Y(r+6)=\frac{r !}{(r+6) !} \sum_{l}^{r} \sum_{s}^{l} \sum_{p}^{s} \frac{(-1)^{p}}{p !} Y(s-p) Y(l-s) Y(r-l)
$$

By using (2.1) at $x=0$, we obtain the following transformed boundary conditions:

$$
Y(0)=1, \quad Y(1)=-\frac{1}{2}, \quad Y(2)=\frac{1}{8}
$$

where $t=y^{\prime \prime \prime}(0) / 3 !=\Upsilon(3), w=y^{(4)}(0) / 4 !=Y(4)$, and $z=y^{(5)}(0) / 5 !=\Upsilon(5)$.

By using the transformed equation (3.36) together with the transformed boundary conditions (3.37), we can easily solve $Y(r)$, for $r \geq 6$. The constants $t$ and $w$ can be evaluated by using boundary conditions (3.35) at $x=1$ :

$$
\begin{gathered}
\sum_{r=0}^{20} Y(r)=e^{-1 / 2}, \\
\sum_{r=0}^{20} r Y(r)=\left(-\frac{1}{2}\right) e^{-1 / 2}, \\
\sum_{k=0}^{20} r(r-1) Y(r)=\left(\frac{1}{4}\right) e^{-1 / 2} .
\end{gathered}
$$

These equations give $t=-0.02083333265, w=0.2604165679 E-2$, and $z=-0.2604149398 E-3$. 
Consequently, the following series solution can be formed by applying the inverse transformation equation in (2.2) up to $N=20$ :

$$
\begin{aligned}
y(x)= & 1.0-0.5 x+0.125 x^{2}-0.2194097232 \times 10^{-1} x^{3} \\
& +0.600832059 \times 10^{-2} x^{4}-0.003832229731 x^{5}+0.001388888889 x^{6} \\
& -0.00009920634921 x^{7}+0.6200396825 \times 10^{-5} x^{8} \\
& -3.994089004 \times 10^{-7} x^{9}+8.476606127 \times 10^{-8} x^{10} \\
& -3.299619968 \times 10^{-8} x^{11}+6.203319625 \times 10^{-9} x^{12} \\
& -2.551978071 \times 10^{-10} x^{13}+3.05519710 \times 10^{-11} x^{14} \\
& -1.574229877 \times 10^{-11} x^{15}+5.71359200 \times 10^{-12} x^{16} \\
& -8.01789691 \times 10^{-13} x^{17}-7.09484742 \times 10^{-14} x^{18} .
\end{aligned}
$$

\section{Adomian Decomposition Method}

Consider the differential equation

$$
L u+R u+N u=g,
$$

where $L$ is invertible and is the highest-order derivative, $R$ is a linear differential operator, where the order of $L$ must be greater than $R, N$ is a nonlinear operator, and $g$ is the source term. By using the given conditions and applying the inverse operator $L^{-1}$ to both sides of (4.1), we get the following equation:

$$
u=f-L^{-1}(R u)-L^{-1}(N u)
$$

where the function $f$ is arising from integrating the source term $g$ and from applying the given conditions which are prescribed.

The series solution of $u(x)$ by the standard Adomian method is given as follows:

$$
u(x)=\sum_{n=0}^{\infty} u_{n}(x)
$$

On the other hand, the nonlinear function $u(x)$ by an infinite series of polynomial is given as follows:

$$
u(x)=\sum_{n=0}^{\infty} A_{n} .
$$


The components $u_{n}$ are determined by using the following relation:

$$
\begin{gathered}
u_{0}=f, \\
u_{k+1}=-L^{-1}\left(R u_{k}\right)-L^{-1}\left(N u_{k}\right)
\end{gathered}
$$

for $k \geq 0$.

From the above equations, we observe that the component $u_{0}$ is identified by the function $f$. We can apply modification by assuming that the function $f$ is divisible into two parts, $f_{0}$ and $f_{1}$. Let the function be as follows:

$$
f=f_{0}+f_{1}
$$

Under this assumption, we have a slight variation for components $u_{0}$ and $u_{1}$, where $f_{0}$ assigned to $u_{0}$ and $f_{1}$ is combined with the other terms in (4.5) to assign $u_{1}$. The modified recursive algorithm is as follows:

$$
\begin{gathered}
u_{0}=f_{0}, \\
u_{1}=f_{1}-L^{-1}\left(R u_{0}\right)-L^{-1}\left(N u_{0}\right), \\
u_{k+2}=-L^{-1}\left(R u_{k+1}\right)-L^{-1}\left(N u_{k+1}\right),
\end{gathered}
$$

for $k \geq 0$. There are several rules that are needed to follow for Adomian polynomials of nonlinear operator $F(u)$ :

$$
\begin{gathered}
A_{0}=F\left(u_{0}\right), \\
A_{1}=u_{1}(x) F^{\prime}\left(u_{0}\right), \\
A_{2}=u_{2} F^{\prime}\left(u_{0}\right)+\frac{u_{1}^{2}}{2 !} F^{\prime \prime \prime}\left(u_{0}\right), \\
A_{3}=u_{3} F^{\prime}\left(u_{0}\right)+u_{1} u_{2} F^{\prime \prime \prime}\left(u_{0}\right)+\frac{u_{1}^{3}}{3 !} F^{\prime \prime \prime}\left(u_{0}\right),
\end{gathered}
$$

and so on; see [3].

For comparison purpose, we solve the boundary value problems in Examples 3.2, 3.3, and 3.4 by using the Adomian decomposition method.

Example 4.1. By using ADM, we solve the following nonlinear equation of order three for fifth-order BVP:

$$
u^{(5)}(x)=e^{-x}(u(x))^{3}, \quad 0<x<1
$$


subject to the boundary conditions

$$
u(0)=1, \quad u^{\prime}(0)=\frac{1}{2}, \quad u^{\prime \prime}(0)=\frac{1}{4}, \quad u(1)=e^{1 / 2}, \quad u^{\prime}(1)=\left(\frac{1}{2}\right) e^{1 / 2}
$$

Equation (4.10) can be rewritten in operator form as follows:

$$
L u=e^{-x}(u(x))^{3}, \quad 0<x<1 .
$$

Operating with fivefold integral operator $L^{-1}$ on (4.12) and using the boundary conditions at $x=0$, we obtain the following equation:

$$
u(x)=1+\frac{1}{2} x+\frac{1}{8} x^{2}+\frac{1}{6} A x^{3}+\frac{1}{24} B x^{4}+L^{-1}\left(e^{-x} u^{3}(x)\right) .
$$

Then, determine the constants $u^{\prime \prime \prime}(0)=A, u^{(4)}(0)=B$. Substituting the decomposition series (4.3) for $u(x)$ and the series of polynomials (4.4) into (4.13) yields

$$
\sum_{n=0}^{\infty} u_{n}(x)=1+\frac{1}{2} x+\frac{1}{8} x^{2}+\frac{1}{6} A x^{3}+\frac{1}{24} B x^{4}+L^{-1}\left(e^{-x} \sum_{n=0}^{\infty} A_{n}\right) .
$$

Then, we split the terms into two parts which are assigned to $u_{0}(x)$ and $u_{1}(x)$ that are not included under $L^{-1}$ in (4.14). We can obtain the following recursive relation:

$$
\begin{gathered}
u_{0}(x)=1 \\
u_{1}(x)=\frac{1}{2} x+\frac{1}{8} x^{2}+\frac{1}{6} A x^{3}+\frac{1}{24} B x^{4}+L^{-1}\left(A_{0}\right), \\
u_{k+1}(x)=L^{-1}\left(A_{k}\right), \quad k \geq 1 .
\end{gathered}
$$

To determine the constants $A$ and $B$, we use the boundary conditions in (4.11) at $x=1$ on the four-term approximant $\phi_{4}$, where

$$
\phi_{4}=\sum_{k=0}^{k=3} y_{k}
$$

Then, solving the above equation yields

$$
A=0.376764, \quad B=-2.154638929 .
$$


Then we get the series solution as follows:

$$
\begin{aligned}
y(x)= & 1.0+0.5 x+0.125 x^{2}+0.6279 \times 10^{-1} x^{3}-0.8977662204 \times 10^{-1} x^{4} \\
& +0.583333333 \times 10^{-1} x^{5}-0.6944444444 \times 10^{-2} x^{6} \\
& -0.0005456349206 x^{7}-0.0001800740079 x^{8}+0.673748941 \times 10^{-4} x^{9} \\
& -0.3295218362 \times 10^{-4} x^{10}+0.741814372 \times 10^{-5} x^{11} \\
& -0.00000119022282 x^{12}-7.604880242 \times 10^{-8} x^{13} \\
& -1.306718703 \times 10^{-10} x^{14}
\end{aligned}
$$

Example 4.2. Now, we solve the following fourth-order nonlinear for fifth-order boundary value problems by using ADM:

$$
u^{(5)}(x)=e^{x}(u(x))^{4}, \quad 0<x<1
$$

subject to the boundary conditions

$$
u(0)=1, \quad u^{\prime}(0)=-\frac{1}{3}, \quad u^{\prime \prime}(0)=\frac{1}{9}, \quad u(1)=e^{-1 / 3}, \quad u^{\prime}(1)=\left(-\frac{1}{3}\right) e^{-1 / 3} .
$$

Equation (4.19) can be rewritten in operator form as follows:

$$
L u=e^{x}(u(x))^{4}, \quad 0<x<1 .
$$

Operating with fivefold integral operator $L^{-1}$ on (4.21) and using the boundary conditions at $x=0$, we obtain the following equation:

$$
u(x)=1-\frac{1}{3} x+\frac{1}{18} x^{2}+\frac{1}{6} A x^{3}+\frac{1}{24} B x^{4}+L^{-1}\left(e^{-x} u^{4}(x)\right)
$$

Then, determine the constants $u^{\prime \prime \prime}(0)=A, u^{(4)}(0)=B$. Substituting the decomposition series (4.3) for $u(x)$ and the series of polynomials (4.4) into (4.22) yields

$$
\sum_{n=0}^{\infty} u_{n}(x)=1-\frac{1}{3} x+\frac{1}{18} x^{2}+\frac{1}{6} A x^{3}+\frac{1}{24} B x^{4}+L^{-1}\left(e^{-x} \sum_{n=0}^{\infty} A_{n}\right) .
$$


Then, we split the terms into two parts which are assigned to $u_{0}(x)$ and $u_{1}(x)$ that are not included under $L^{-1}$ in (4.23). We can obtain the following recursive relation:

$$
\begin{gathered}
u_{0}(x)=1, \\
u_{1}(x)=-\frac{1}{3} x+\frac{1}{18} x^{2}+\frac{1}{6} A x^{3}+\frac{1}{24} B x^{4}+L^{-1}\left(A_{0}\right), \\
u_{k+1}(x)=L^{-1}\left(A_{k}\right), \quad k \geq 1 .
\end{gathered}
$$

To determine the constants $A$ and $B$, we use the boundary conditions in (4.20) at $x=1$ on the four-term approximant $\phi_{4}$, where

$$
\phi_{5}=\sum_{k=0}^{k=4} y_{k}
$$

Then, solving the above equation yields

$$
A=-1.178489258, \quad B=-0.1205488584 .
$$

Finally, we get the series solution as follows:

$$
\begin{aligned}
y(x)= & 1-0.3333333333 x+0.05555555556 x^{2}-0.4453076220 x^{3} \\
& -0.4040993754 \times 10^{-1} x^{4}+0.84845560 x^{5}-0.298115585 x^{6} \\
& -0.680956807 \times 10^{-1} x^{7}-0.2456515229 \times 10^{-2} x^{8} \\
& +0.6206348264 \times 10^{-3} x^{9}-0.1181956121 \times 10^{-3} x^{10} \\
& -0.0001615659149 x^{11}-0.7466165099 \times 10^{-4} x^{12} \\
& -0.2012942457 \times 10^{-4} x^{13}-0.6094555007 \times 10^{-5} x^{14} \\
& -0.1080643342 \times 10^{-5} x^{15}-8.736244687 \times 10^{-8} x^{16} \\
& -1.417232124 \times 10^{-9} x^{17}+2.178184252 \times 10^{-11} x^{18} .
\end{aligned}
$$

Example 4.3. Finally, we perform third order of nonlinear function for sixth-order BVP for ADM as follows:

$$
u^{(6)}(x)=e^{x}(u(x))^{3}, \quad 0<x<1
$$


subject to the boundary conditions

$$
\begin{gathered}
u(0)=1, \quad u^{\prime}(0)=-\frac{1}{2}, \quad u^{\prime \prime}(0)=\frac{1}{4}, \quad u(1)=e^{-1 / 2}, \\
u^{\prime}(1)=\left(-\frac{1}{2}\right) e^{-1 / 2}, \quad u^{\prime \prime}(1)=\left(\frac{1}{4}\right) e^{-1 / 2} .
\end{gathered}
$$

Equation (4.28) can be rewritten in operator form as follows:

$$
L u=e^{-x}(u(x))^{3}, \quad 0<x<1 .
$$

Operating with sixfold integral operator $L^{-1}$ on (4.30) and using the boundary conditions at $x=0$, we obtain the following equation:

$$
u(x)=1-\frac{1}{2} x+\frac{1}{8} x^{2}+\frac{1}{6} A x^{3}+\frac{1}{24} B x^{4}+\frac{1}{120} C x^{5}+L^{-1}\left(e^{-x} u^{4}(x)\right)
$$

Then, determine the constants $u^{\prime \prime \prime}(0)=A, u^{(4)}(0)=B$, and $u^{(5)}(0)=C$. Substituting the decomposition series (4.3) for $u(x)$ and the series of polynomials (4.4) into (4.31) yields

$$
\sum_{n=0}^{\infty} u_{n}(x)=1-\frac{1}{2} x+\frac{1}{8} x^{2}+\frac{1}{6} A x^{3}+\frac{1}{24} B x^{4}+\frac{1}{120} C x^{5}+L^{-1}\left(e^{-x} \sum_{n=0}^{\infty} A_{n}\right) .
$$

Then similarly, we split the terms into two parts which are assigned to $u_{0}(x)$ and $u_{1}(x)$ that are not included under $L^{-1}$ in (4.32). We can obtain the following recursive relation:

$$
\begin{gathered}
u_{0}(x)=1, \\
u_{1}(x)=-\frac{1}{2} x+\frac{1}{8} x^{2}+\frac{1}{6} A x^{3}+\frac{1}{24} B x^{4}+\frac{1}{120} C x^{5}+L^{-1}\left(A_{0}\right) \\
u_{k+1}(x)=L^{-1}\left(A_{k}\right), \quad k \geq 1 .
\end{gathered}
$$

To determine the constants $A, B$, and $C$, we use the boundary conditions in (4.29) at $x=1$ on the four-term approximant $\phi_{4}$, where

$$
\phi_{4}=\sum_{k=0}^{k=3} y_{k}
$$

Then, solving the above equation yields

$$
A=-14.69918030, \quad B=102.1542319, \quad C=-219.2146156 .
$$


Table 1: Numerical result for Example 3.2 and Example 4.1.

\begin{tabular}{lccc}
\hline$x$ & Exact solution & DTM $(N=14)$ & ADM $(N=14)$ \\
\hline 0.0 & 1 & 1 & 1 \\
0.1 & 1.051271096 & 1.051278920 & 1.051304392 \\
0.2 & 1.105170918 & 1.105220776 & 1.105376925 \\
0.3 & 1.161834243 & 1.161964144 & 1.162354804 \\
0.4 & 1.221402759 & 1.221630872 & 1.222288426 \\
0.5 & 1.284025416 & 1.284337420 & 1.285197759 \\
0.6 & 1.284025416 & 1.284337420 & 1.351122659 \\
0.7 & 1.284025416 & 1.284337420 & 1.420166655 \\
0.8 & 1.284025416 & 1.284337420 & 1.492533672 \\
0.9 & 1.284025416 & 1.284337420 & 1.568557204 \\
1.0 & 1.284025416 & 1.284337420 & 1.648721285 \\
\hline
\end{tabular}

Table 2: Numerical result for Example 3.3 and Example 4.2.

\begin{tabular}{lccc}
\hline$x$ & Exact solution & DTM $(N=18)$ & ADM $(N=18)$ \\
\hline 0.0 & 1 & 1 & 1 \\
0.1 & 0.9672161006 & 0.9672221453 & 0.9667810534 \\
0.2 & 0.9355069849 & 0.9672221453 & 0.9321799874 \\
0.3 & 0.9048374181 & 0.9049350590 & 0.8944787522 \\
0.4 & 0.8751733191 & 0.8753424236 & 0.8533754418 \\
0.5 & 0.8464817250 & 0.8467098288 & 0.8103487343 \\
0.6 & 0.8187307532 & 0.8189816371 & 0.7687007110 \\
0.7 & 0.7918895662 & 0.7921124409 & 0.7332405365 \\
0.8 & 0.7659283385 & 0.7660753950 & 0.7095695543 \\
0.9 & 0.7408182206 & 0.7408702826 & 0.7029247972 \\
1.0 & 0.7165313107 & 0.7165313107 & 0.7165313263 \\
\hline
\end{tabular}

Finally, we get the series solution as follows:

$$
\begin{aligned}
y(x)= & 1-0.5 x+0.125 x^{2}-2.449863383 x^{3}+4.256426329 x^{4} \\
& -1.826788463 x^{5}+0.138888888 \times 10^{-2} x^{6}+0.1984126984 \times 10^{-3} x^{7} \\
& +0.0000248015873 x^{8}+0.2292633427 \times 10^{-3} x^{9}-0.343791062 \times 10^{-4} x^{10} \\
& -0.680212248 \times 10^{-4} x^{11}+0.406878271 \times 10^{-4} x^{12} \\
& -0.00005031070540 x^{13}+0.00003795993196 x^{14}-0.128793671 \times 10^{-4} x^{15} \\
& +0.000001752215748 x^{16}-5.518698119 \times 10^{-12} x^{17} .
\end{aligned}
$$

\section{Results}

We provide the results of the given examples in Tables 1, 2, and 3. 
Table 3: Numerical result for Example 3.4 and Example 4.3.

\begin{tabular}{lccc}
\hline$x$ & Exact solution & DTM $(N=18)$ & ADM $(N=18)$ \\
\hline 0.0 & 1 & 1 & 1 \\
0.1 & 0.9512294245 & 0.9492075127 & 0.9492075127 \\
0.2 & 0.9048374181 & 0.8916268943 & 0.8916268943 \\
0.3 & 0.8607079765 & 0.8251427077 & 0.8251427077 \\
0.4 & 0.8187307532 & 0.7534730280 & 0.7534730280 \\
0.5 & 0.7788007831 & 0.6839803183 & 0.6839803183 \\
0.6 & 0.7408182206 & 0.6254839642 & 0.6254839642 \\
0.7 & 0.7046880897 & 0.7046783358 & 0.5860748693 \\
0.8 & 0.6703200461 & 0.6703157625 & 0.5709325210 \\
0.9 & 0.6376281517 & 0.6376273947 & 0.5801448253 \\
1.0 & 0.6065306598 & 0.6065306599 & 0.6065306590 \\
\hline
\end{tabular}

\section{Conclusion}

From the results, the proposed method, as well as the Differential Transformation Method, is more accurate than the Adomian Decomposition Method. The errors between the solutions of Differential Transformation Method and the exact solutions are smaller compared to the errors between the solutions of Adomian Decomposition Method and the exact solutions. In addition, Differential Transformation Method also shows less computational effort because it needs less time in calculation. Besides that, it is hard to calculate Adomian polynomials. From the results we obtained, it can reinforce conclusion made by many researchers that Differential Transformation Method is more efficient and accurate than Adomian Decomposition Method. Therefore, we can conclude that Differential Transformation Method is applicable for such problems in the bounded domains. The computations in all examples were performed by using Maple 13 .

\section{Acknowledgments}

The authors gratefully acknowledge that this research was partially supported by the University Putra Malaysia under the Research University Grant scheme 05-01-09-0720RU and Fundamental Research Grant scheme 01-11-09-723FR. The authors also thank the referee(s) for very constructive comments and suggestions that improved the paper.

\section{References}

[1] A.-M. Wazwaz, "A reliable modification of Adomian decomposition method," Applied Mathematics and Computation, vol. 102, no. 1, pp. 77-86, 1999.

[2] A.-M. Wazwaz, "Approximate solutions to boundary value problems of higher order by the modified decomposition method," Computers \& Mathematics with Applications, vol. 40, no. 6-7, pp. 679-691, 2000.

[3] A.-M. Wazwaz, "The numerical solution of fifth-order boundary value problems by the decomposition method," Journal of Computational and Applied Mathematics, vol. 136, no. 1-2, pp. 259-270, 2001.

[4] A.-M. Wazwaz, "The numerical solution of sixth-order boundary value problems by the modified decomposition method," Applied Mathematics and Computation, vol. 118, no. 2-3, pp. 311-325, 2001.

[5] M. Meštrović, "The modified decomposition method for eighth-order boundary value problems," Applied Mathematics and Computation, vol. 188, no. 2, pp. 1437-1444, 2007. 
[6] M. M. Hosseini and M. Jafari, "A note on the use of Adomian decomposition method for highorder and system of nonlinear differential equations," Communications in Nonlinear Science and Numerical Simulation, vol. 14, pp. 1952-1957, 2009.

[7] M. Dehghan and F. Shakeri, "The numerical solution of the second Painlevé equation," Numerical Methods for Partial Differential Equations, vol. 25, no. 5, pp. 1238-1259, 2009.

[8] M. Dehghan and M. Tatari, "The use of Adomian decomposition method for solving problems in calculus of variations," Mathematical Problems in Engineering, vol. 2006, Article ID 65379, 9 pages, 2006.

[9] M. Dehghan and R. Salehi, "A seminumeric approach for solution of the eikonal partial differential equation and its applications," Numerical Methods for Partial Differential Equations, vol. 26, no. 3, pp. 702-722, 2010.

[10] M. Dehghan, J. M. Heris, and A. Saadatmandi, "Application of semi-analytic methods for the Fitzhugh-Nagumo equation, which models the transmission of nerve impulses," Mathematical Methods in the Applied Sciences, vol. 33, no. 11, pp. 1384-1398, 2010.

[11] M. Dehghan, M. Shakourifar, and A. Hamidi, "The solution of linear and nonlinear systems of Volterra functional equations using Adomian-Pade technique," Chaos, Solitons and Fractals, vol. 39, no. 5, pp. 2509-2521, 2009.

[12] M. Dehghan, A. Hamidi, and M. Shakourifar, "The solution of coupled Burgers' equations using Adomian-Pade technique," Applied Mathematics and Computation, vol. 189, no. 2, pp. 1034-1047, 2007.

[13] M. Dehghan and R. Salehi, "Solution of a nonlinear time-delay model in biology via semi-analytical approaches," Computer Physics Communications, vol. 181, no. 7, pp. 1255-1265, 2010.

[14] F. Shakeri and M. Dehghan, "Application of the decomposition method of adomian for solving the pantograph equation of order m," Zeitschrift fur Naturforschung, vol. 65, no. 5, pp. 453-460, 2010.

[15] M. Dehghan, "The solution of a nonclassic problem for one-dimensional hyperbolic equation using the decomposition procedure," International Journal of Computer Mathematics, vol. 81, no. 8, pp. 979 989, 2004.

[16] S. S. Ray and R. K. Bera, "Analytical solution of a fractional diffusion equation by Adomian decomposition method," Applied Mathematics and Computation, vol. 174, no. 1, pp. 329-336, 2006.

[17] F. Ayaz, "On the two-dimensional differential transform method," Applied Mathematics and Computation, vol. 143, no. 2-3, pp. 361-374, 2003.

[18] F. Ayaz, "Solutions of the system of differential equations by differential transform method," Applied Mathematics and Computation, vol. 147, no. 2, pp. 547-567, 2004.

[19] V.S. Ertürk and S. Momani, "Comparing numerical methods for solving fourth-order boundary value problems," Applied Mathematics and Computation, vol. 188, no. 2, pp. 1963-1968, 2007.

[20] A. Arikoglu and I. Ozkol, "Solution of fractional differential equations by using differential transform method," Chaos, Solitons and Fractals, vol. 34, no. 5, pp. 1473-1481, 2007.

[21] I. H. Abdel-Halim Hassan and V. S. Ertürk, "Solutions of different types of the linear and non-linear higher-order boundary value problems by differential transformation method," European Journal of Pure and Applied Mathematics, vol. 2, no. 3, pp. 426-447, 2009. 


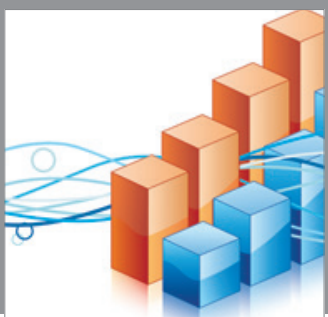

Advances in

Operations Research

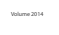

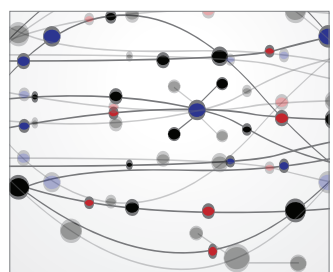

\section{The Scientific} World Journal
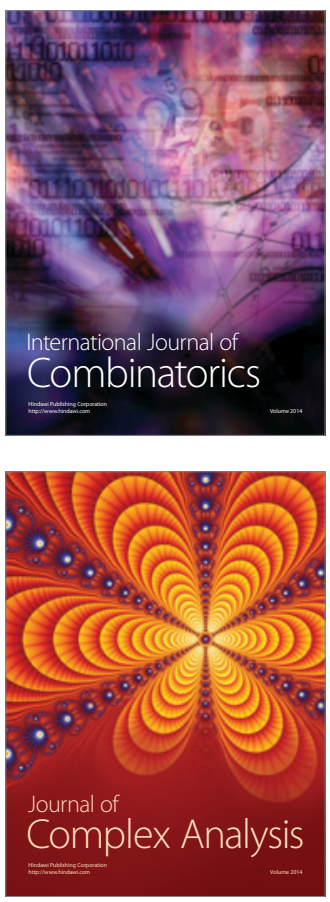

International Journal of

Mathematics and

Mathematical

Sciences
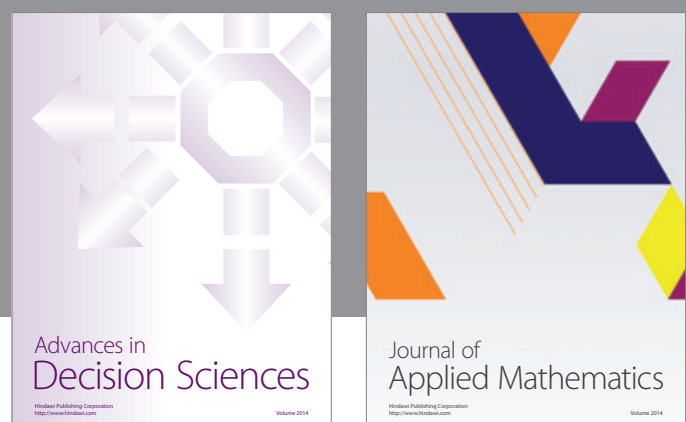

Journal of

Applied Mathematics
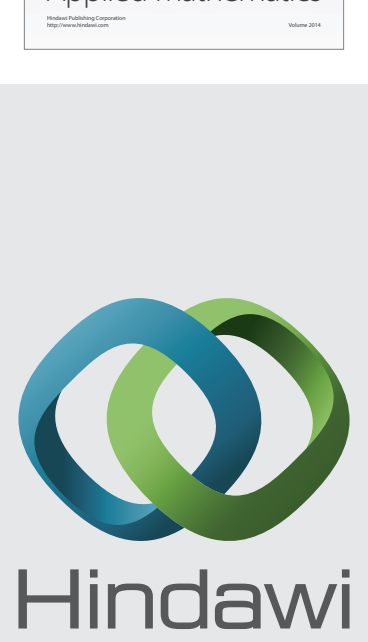

Submit your manuscripts at http://www.hindawi.com
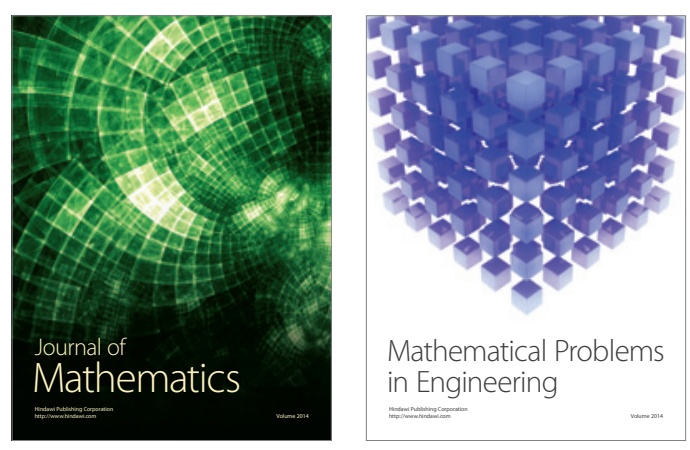

Mathematical Problems in Engineering
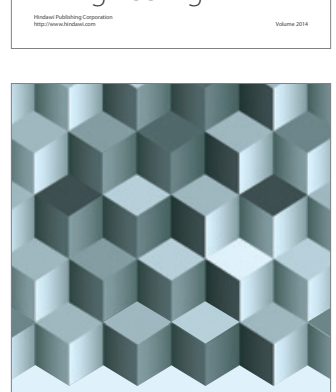

Journal of

Function Spaces
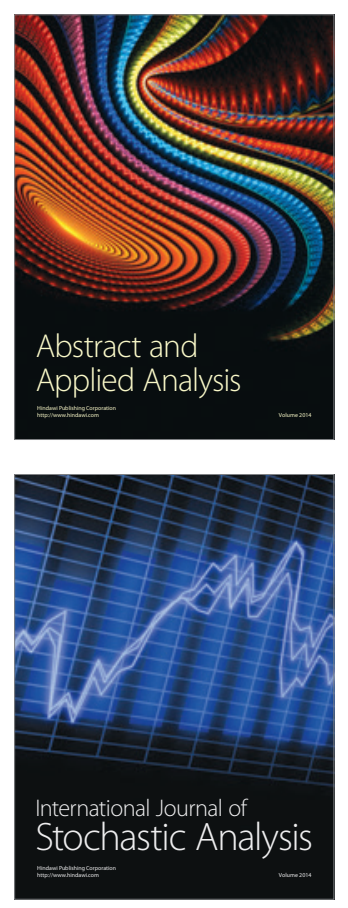

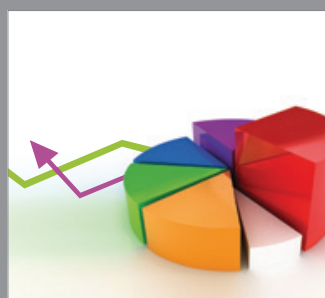

ournal of

Probability and Statistics

Promensencen
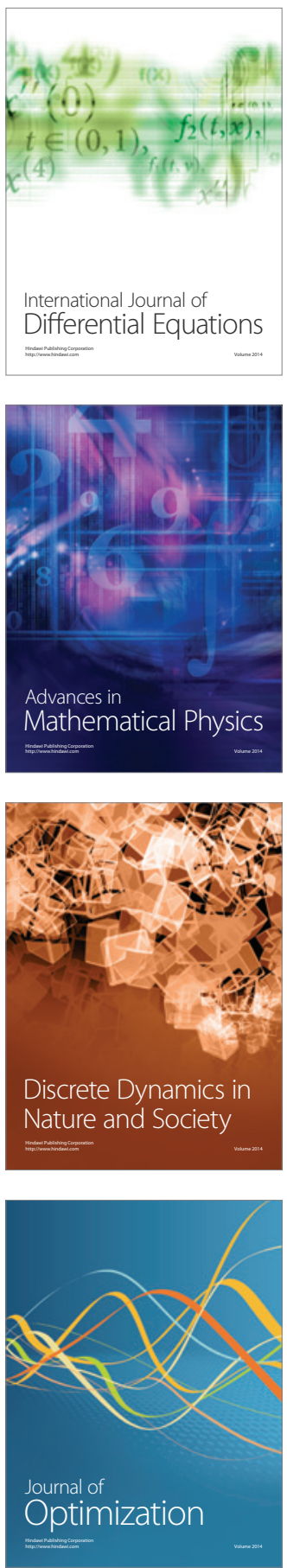